\title{
A Problem Oriented Approach to Data Mining in Distributed Spatio-temporal Database
}

\author{
Zhou Huang, Yu Fang, Xia Peng, Bin Chen, and Xuetong Xie \\ Institute of Remote Sensing \& GIS, Peking University \\ Beijing, P.R. China 100871 \\ huangzhou@pku.edu.cn
}

\begin{abstract}
Recently, a fast increment of spatio-temporal data volume has been achieved and more importantly the data might distribute everywhere. So, there is a need for spatio-temporal data mining systems that are able to support such distributed spatio-temporal query and analysis operations. Distributed spatiotemporal data mining technologies were discussed in this paper. After discussing the process of spatio-temporal data mining in distributed environment, one actual DSTDMS (Distributed Spatio-Temporal Data Mining System) was designed and then implemented. The system is based on data model of sequent snapshot and accomplished through spatio-temporal extension on PostgreSQL. Various spatio-temporal analyses and mining queries could be carried out in the system through simple SQL statements. By using the system, effective mining of distributed spatio-temporal data were achieved.
\end{abstract}

Keywords: Spatio-temporal data, Data mining, Distributed database, GIS.

\section{Introduction}

Along with the wide use of information technology, more and more attention is being paid on spatio-temporal data [1]. In GIS (Geographic Information System) research realm, how to discover useful knowledge among huge data distributed in network is one of the most concerned topics. There are many related researches now. Erwig [1] had a comparison and analysis among those common spatio-temporal data models, including sequent snapshot model, base state with amendment model, event based model, object oriented model and so on. Hamilton [2] concluded the architecture of distributed spatio-temporal database system and defined the concept of STDML (Spatio-Temporal Data Mining Language) and STDM (Spatio-Temporal Data Model) as well. Harms [3] researched on the architecture of knowledge discovery in spatiotemporal database. Pokrajac [4] indicated that a well approach to STDML would be achieved by extending SQL (Structured Query Language) for supporting spatiotemporal operations.

Existing researches mainly concentrate on STDM, STDML, Spatio-temporal mining algorithms, etc. However, a chief problem is that there is not one common and effective method for accomplishing a well-formed spatio-temporal data mining system, especially in the circumstance of distributed spatio-temporal database [5]. 
Complex characteristics of STDM and STDML, as well as lack of unified distributed spatio-temporal database architecture are the main reasons to that problem. So, it is necessary for us to provide a common and effective method for constructing DSTDMS. That is also a nature trend in GIS realm and of significance to promote the application based on spatio-temporal data.

Basing on key technologies of distributed spatio-temporal database, this paper concluded the structure model of DSTDMS (Distributed Spatio-Temporal Data Mining System). Then an instance of DSTDMS named SpatialMiningService was achieved based on that model. This paper accomplished the prototype by using PostgreSQL as the backend database and sequent snapshot as spatio-temporal data model. Running effect indicates that the system provides an effective way for the spatio-temporal data mining and analyses.

\section{Structure Model of DSTDMS}

Before discussing the data mining in distributed spatio-temporal database, the spatiotemporal data management should be referred firstly, which relates close with the data mining approach. Spatio-temporal data is unique for its spatial and temporal elements. The traditional method for GIS data management_ combination of file and relation database__ is not suitable for spatio-temporal data management [5]. And, object oriented database system is still a long way to actual applications. So, an appropriate approach is adopting ORDB (Object-Relation Database) to store and manipulate the spatio-temporal data [6]. Moreover, Spatio-temporal extension on ORDB is needed

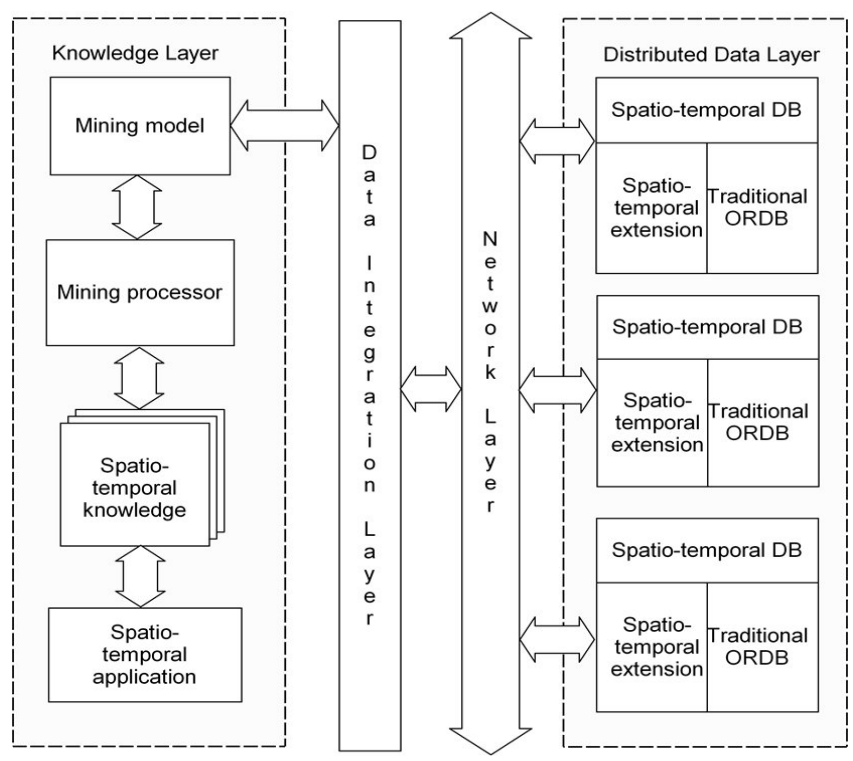

Fig. 1. Structure model of DSTDMS 
for supporting operations on spatial and temporal elements and many mainstream ORDB products like Oracle, Informix and PostgreSQL all provide the mechanism of spatio-temporal extension. Thus, a typical and practical distributed spatio-temporal database is composed of a set of object-relation databases, on which spatio-temporal extension would be accomplished.

Based on the discussion above, the typical structure of DSTDMS based on ORDB backend is shown in Fig.1. Three layers, including knowledge layer, data integration layer and spatio-temporal database layer, construct a complete DSTDMS.

(1)Distributed data layer. The backend layer is composed of a set of separated spatio-temporal databases distributed on several data sites. This layer is used for spatio-temporal data storage and the data distribution could be redundant to obtain higher efficiency and stability [7]. Usually one common ORDB product like PostgreSQL or Oracle would be adopted. The capabilities of supporting spatiotemporal extension and SQL query are indispensable.

(2)Data integration layer. The middle layer in the model mainly refers to preselecting the data needed from separated data sites and integrating them into one complete view. This step is crucial for spatio-temporal discovery as it makes up of the data basis for further mining. This layer accepts global analysis query and translates it into a series of sub-queries, often in form of SQL statements. Then the sub-queries would be executed on selected sites and the final global data view is to be generated. Currently this topic is seldom referred in the researches of distributed spatio-temporal data mining, so this paper paid more attention on it and detailed distributed query processing algorithms were brought out and demonstrated in the system design section below.

(3)Knowledge layer. This layer is on the top of structure model and composed of various applications to abstract the specified knowledge from the spatio-temporal data, such as data visualization, classification, association, fitting, professional modeling, scientific computation, prediction and so on. The components of this layer mainly contain Mining processor and various spatio-temporal applications. The top layer is application oriented and various mining algorithms have been developed and discussed fitting to the specified spatio-temporal applications [8].

\section{Design and Implementation}

Based on the researches on distributed spatio-temporal data mining system model, the authors implemented an actual DSTDMS named SpatialMiningService. The system architecture was shown as Fig.2, in which the implementation could be concluded into two parts, including spatio-temporal data model, spatio-temporal extension and distributed query processing.

Query language this system used is adopted language compatible with SQL completely, so developers need not to do extra work as the system was extended on ORDB. Spatio-temporal extension should be done after adopting one suitable data 


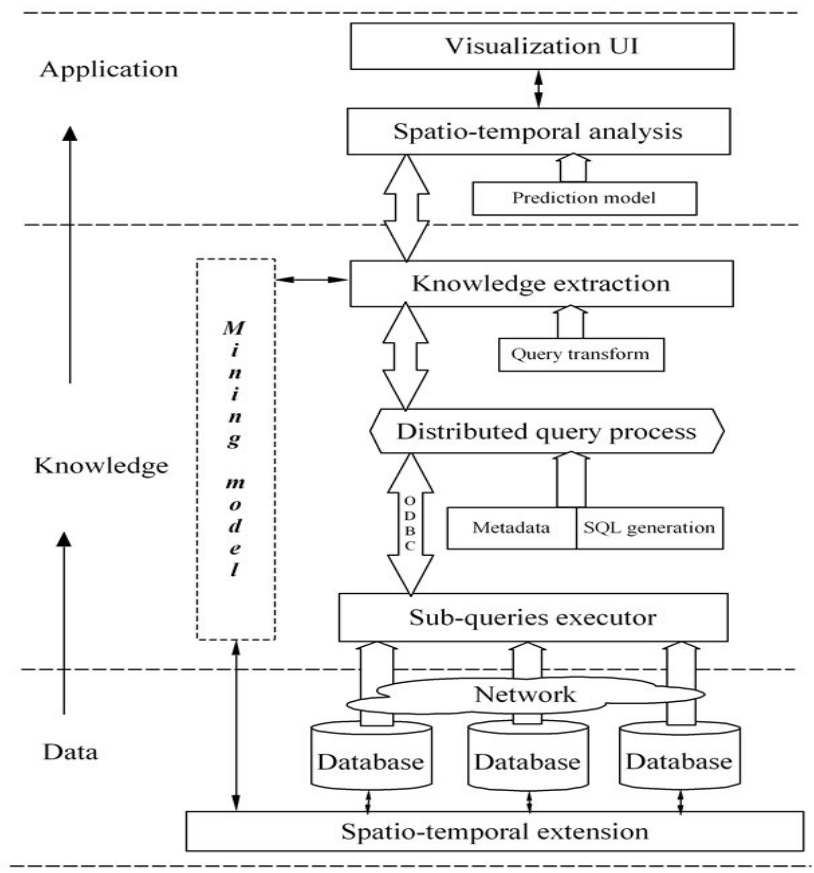

Fig. 2. System architecture of SpatialMiningService

model. SpatialMiningService uses sequent snapshot model and stores some corresponding sample data for test.

\subsection{Sequent Snapshot Model}

To describe the spatial objects two data models are often referred, vector model and raster model, which are also called feature model or domain model. The vector structure is used to express the discrete and separated objects while the raster structure are declined to describe the continuous phenomenon [9]. The raster model has a wide application on temperature, rainfall, pollution distribution, population evaluation, etc. Current raster model mainly refers to static model, which means it has no temporal dimension and could not express the spatio-temporal phenomenon. Considering it this paper adopted one model taking temporal dimension into account, which was called sequent snapshot model.

Sequent snapshot can be defined as $S S M=(M, S, T)$, where: $S$ and $T$ respsent the spatial and temporal variables. The spatial domain is partitioned into regularly rectangular grid, each of which has one value or intensity. The grids are represented by matrix. In temporal domain, a period was discretized according to a given size and divided into a series of moments. At every moment, the varying phenomenon was defined as a snapshot which was represented as a matrix with values. $M$ is a 4 -d array like $M[x, y, z, t]$, which consists of a group of matrixes in 
temporal order, usually in the form of $m_{1}, m_{2}, \ldots, m_{i}, \ldots, m_{n}(1 \leq i \leq n)$, where $\left\{t_{1}, t_{2}, \ldots, t_{i}, \ldots, t_{n}\right\} \subset T, m_{i}$ is the matrix snapshot at the moment $t_{i}$.

\subsection{Spatio-temporal Extension}

This paper extended sequent snapshot type and corresponding spatio-temporal operations based on PostgreSQL database. The type structure was shown in Table.1 and extra temporal type should be extended to record and represent the temporal variable.

Table 1. Demonstration of sequent snapshot type extension

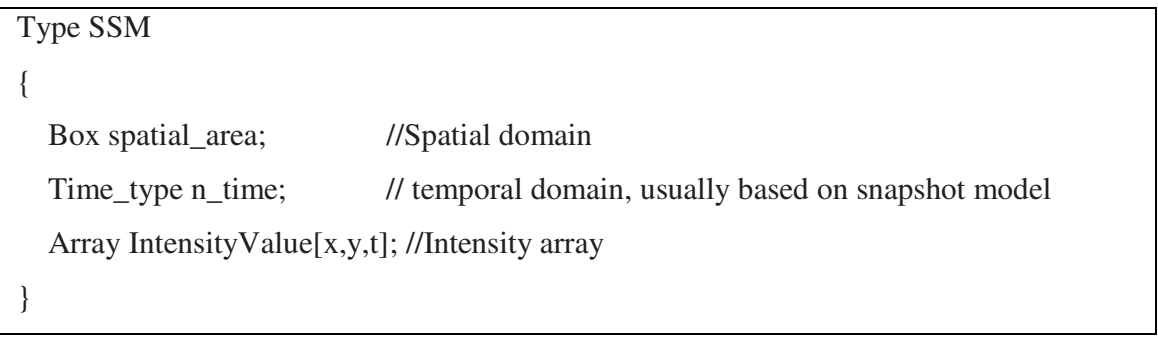

Mining model extension was accomplished in the system as well. Those mainly refer to extend spatio-temporal analysis functions between different spatio-temporal objects or data sources, such as spatial overlap function, spatial-temporal aggregation function, etc. Thus, mining process could be achieved through simple SQL statements, in which using the extended mining functions. So, the users only have to know the "problem" and submit one SQL statement and then the final knowledge would return. That's why the approach this paper brought up is called "problem oriented".

\subsection{Distributed Query Processing}

In SpatialMiningService, distributed query processing algorithm was combined the heuristic principles with the semi-join strategy. We concluded five basic heuristic rules in the distributed spatio-temporal query processing: (1)Execute the pre-select operations as soon as possible; (2)Execute the project operations as soon as possible; (3)Avoid only execute the Descartes accumulate but combination with selection and project operations; (4) Make the account of parallel operations most; Select the strategy of less data transmission.

Based on the heuristic rules aforementioned and the characteristics of spatiotemporal query, the authors brought out one novel algorithm named HHOA (Hybrid Heuristic Optimization Algorithm), which is briefly described as follows. 
Table 2. HHOA algorithm

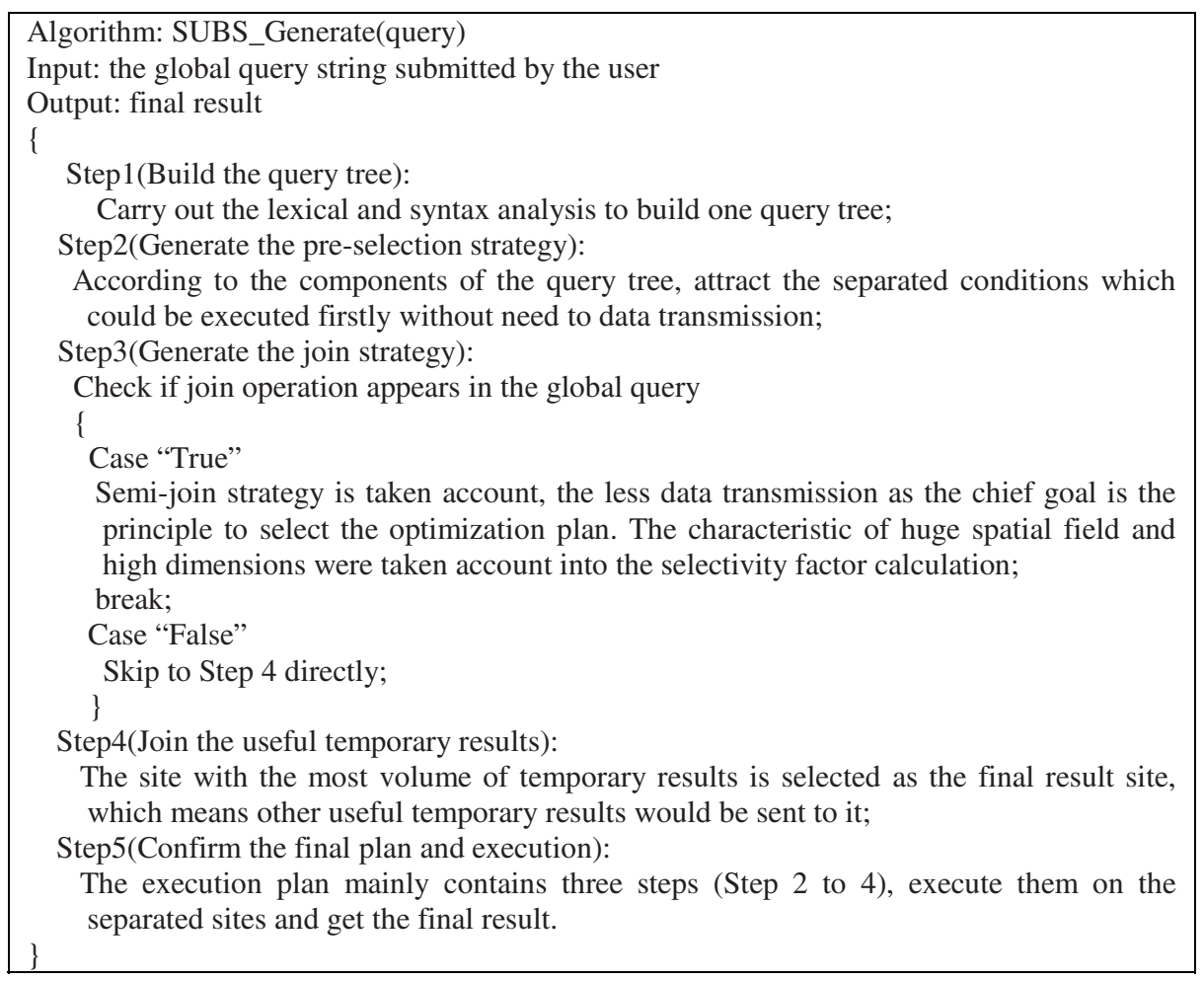

\section{Discussion}

For demonstrating the application of SpatialMiningService the authors collected and input some sampling line and polygon data into the system, partitioning the complete data view into several ones according to the spatial domain and distributing them onto different sites (In fact, we used the railway and county data of China at different

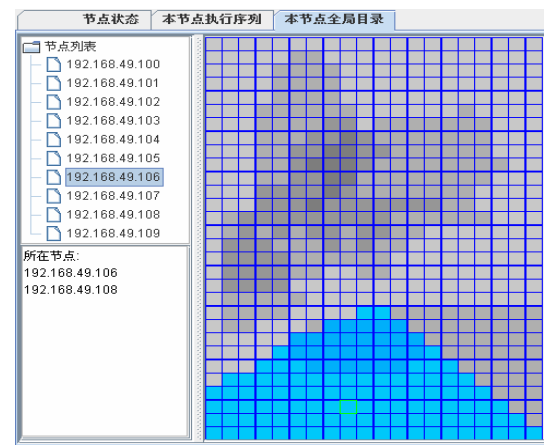

Fig. 3. The sampling data distribution 
temporal points). The sampling data distribution could be shown as Fig.3 (Friendly visualization user interface was accomplished by the authors as well, through which the users could see the data distribution, knowledge visualization and even the status of the sub-queries in the execution plan).

Then the users could use simple SQL statement to model the discrete data or obtain advanced spatio-temporal knowledge. For example, we used this statement "select county.name, county.geometry from county, rail where Overlap(rail.geometry, county.geometry) and rail.name='Zhegang Railway' and rail.time $=$ '1997'" to abstract the counties which the Zhegang Railway cross through at 1997. The returned result is graphically expressed as Fig.4. Also, clearly we obtained the complete data and knowledge view from the distributed spatio-temporal database according to the result.

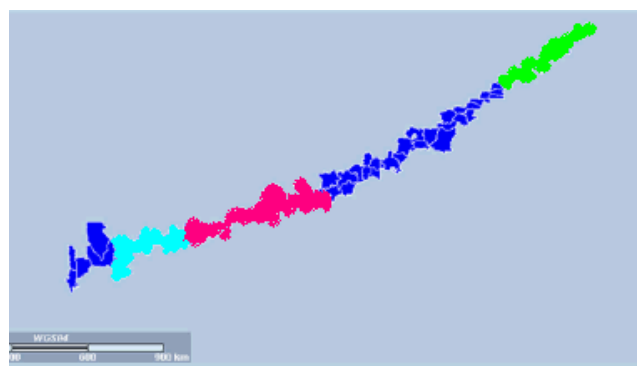

Fig. 4. The sample mining query result (different colors represent different data sites)

\section{Conclusion}

Spatio-temporal data mining system based on distributed database was implemented through the distributed query processing and spatio-temporal extension, mainly containing type, operation and mining model extension. This paper used the data model of sequent snapshot and constructed one actual DSTDMS named SpatialMiningService. Some experiments had been done and running effect indicates that the system provides ability for spatio-temporal manipulation and advanced analysis. Furthermore, more complex applications based on the system or this method should be experimented and more intelligent mining query language should be taken into account.

\section{Acknowledgments}

This research was supported by the following grants from the NSFC Project (40501052) and the Survey and Mapping Foundation in 2005.

\section{References}

1. Erwig M., Schneider M. and Guting R.H.: Temporal Objects for Spatio-Temporal Data Models and a Comparison of Their Representations. Proceedings of the Workshops on Data Warehousing and Data Mining (1998) 
2. Hamilton H.J. and Findlater L.: Looking Backward, Forward, and All Around: Temporal, Spatial, and Spatio-Temporal Data Mining. Proceedings of the Fifteenth International Florida Artificial Intelligence Research Society Conference (2002)

3. Harms S.K., Deogun J., Goddard S.: Building knowledge discovery into a geo-spatial decision support system. Proceedings of the 2003 ACM symposium on Applied computing (2003)

4. Pokrajac D., Hoskinson R.L., Obradovic Z.: Modeling spatial-temporal data with a short observation history. Knowledge and Information Systems. 5(3):86-95(2003)

5. Gudmundsson J., Kreveld M., Speckmann B.: Efficient detection of motion patterns in spatio-temporal data sets. Proceedings of the 12th annual ACM international workshop on Geographic information systems (2004)

6. Lin J., Keogh E., Lonardi S.: A symbolic representation of time series, with implications for streaming algorithms. Proceedings of the 8th ACM SIGMOD workshop on Research issues in data mining and knowledge discovery (2003)

7. Aggarwal C.C.: A framework for diagnosing changes in evolving data streams. Proceedings of the 2003 ACM SIGMOD international conference on Management of data (2003)

8. Kitamoto A.: Spatio-Temporal Data Mining for Typhoon Image Collection. Journal of Intelligent Information Systems. 19(1):19-26(2002)

9. Wang Y., Hijikata Y. and Nishida S.: A Study on a Spatio-Temporal Data Structure for Managing Video Data from Monitoring Cameras. Proceedings of the 17th International Conference on Advanced Information Networking and Applications (2003) 